\title{
On the dynamical stability of the Rosetta orbiter. I.
}

\author{
E. Mysen and K. Aksnes
}

\author{
Institute of Theoretical Astrophysics, University of Oslo, PO Box 1029 Blindern, 0315 Oslo, Norway \\ e-mail: [eirik.mysen; kaare.aksnes] @astro.uio.no \\ Received 5 October 2005 / Accepted 11 May 2006
}

\section{ABSTRACT}

\begin{abstract}
The Rosetta probe is to monitor, from a bound orbit, its primary target comet 67P/Churyumov-Gerasimenko for extended periods of time. As a preliminary assessment of the challenges involved, the strengths of the effects which perturb the spacecraft's cometocentric Keplerian trajectory are evaluated. It is found that in our adopted nominal scenario, where the CO outgassing rate is set to one tenth of its upper limit, motion could be considered regular a long time after rendezvous. Furthermore, in this weak limit, gravity perturbations have a negligible destabilizing effect on the shape of the orbit, while the secular impact risk under the effect of radiation pressure is minimized by placing the orbit in the plane normal to the cometocentric direction of the Sun, with a low initial eccentricity. The comet's heliocentric distance for probe escape for an orbit started in the solar plane-of-sky, however, is seen to be dependent on the initial semi-major axis only, and linearly so. In order to calculate the dynamical effects of outgassing, a radially directed, asymmetric and periodically time-varying pressure field is adopted. Accordingly, it is shown that specific field asymmetries, related to the tendency of the outgassing field to "remember" the direction of comet motion, represent the extreme scenarios as far as orbital stability for the full problem is concerned.
\end{abstract}

Key words. celestial mechanics - space vehicles - comets: individual: 67P/Churyumov-Gerasimenko methods: analytical - methods: numerical

\section{Introduction}

In 2014 the Rosetta probe will rendezvous with comet $67 \mathrm{P} /$ Churyumov-Gerasimenko where it is to be inserted into a stable orbit. Nominally, the probe is then to monitor the comet from this bound orbit for extended periods of time. The success of this scenario is dependent on the weakness of the effects which disturb the probe's cometocentric Keplerian trajectory, and the nature of these perturbations. Therefore, we will first present a preliminary analysis of the perturbation strength, and then proceed to discuss the properties of the various perturbations mostly in the weak limit, as is required for extended close observation.

The focus of this study is on the tendencies which are induced by the uncertain intrinsic forces of the system, and the planned and controllable thruster maneuvers are not incorporated. For the same reason, general orbit configurations are considered. The presented analysis therefore serves as a preparation for contingencies, as well as an indication of how much active control is needed for different strategies and objectives.

\section{Equations of motion}

Our chosen reference axes with unit vectors $\boldsymbol{u}_{X_{i}}$, where $X_{i}=X$, $Y$ and $Z$, are defined to originate from the mass center of the comet nucleus, and are assumed not to rotate with respect to a Newtonian inertial system.

\subsection{Gravity}

The gravitational field of the comet nucleus, deviating from the Keplerian potential

$V_{0}=-\frac{\mu_{\mathrm{c}}}{r}$, can be modelled using a spherical harmonics expansion (Heiskanen \& Moritz 1967)

$$
\begin{aligned}
V_{1}= & \frac{\mu_{\mathrm{c}}}{r} \sum_{n=2}^{\infty} \sum_{m=0}^{n}\left(\frac{r_{\mathrm{c}}}{r}\right)^{n} P_{n m}(\sin \beta) \\
& \times\left[c_{n m} \cos m \lambda+s_{n m} \sin m \lambda\right],
\end{aligned}
$$

resulting in the total gravitational mass center acceleration of a nearby body

$\ddot{\boldsymbol{r}}=-\nabla\left(V_{0}+V_{1}\right), \quad \nabla \equiv \sum_{i=1}^{3} \boldsymbol{u}_{X_{i}} \frac{\partial}{\partial X_{i}}$.

Above $\mu_{\mathrm{c}}$ is the product of the gravitational constant and the comet mass, $r$ is the distance of the probe from the comet's mass center, while $\lambda$ and $\beta$ are the spacecraft's cometocentric east longitude and north latitude, respectively, relative to the nucleus' principal axes (Goldstein 1980). In a principal axis system with origin at the mass center of the comet, only the $c_{n 0}$ and $c_{n 2}$ coefficients are different from zero if $n=2$. The reference radius $r_{\mathrm{c}}$ is usually set equal to the largest dimension of the body. Spherical harmonics have been Fourier expanded in the nucleus attitude angles by Kinoshita et al. (1974), making analytic studies feasible for the general case of excited nucleus rotation.

In addition, the spacecraft is affected by the gravity from the Sun which, relative to the comet, is countered by the heliocentric acceleration of the nucleus. Under the fair assumption of two-body comet heliocentric motion, the result is a differential acceleration

$\ddot{\boldsymbol{r}}=\frac{2 \mu_{\odot}}{R^{3}}\left(\boldsymbol{u}_{\odot} \cdot \boldsymbol{r}\right) \boldsymbol{u}_{\odot}, \quad V_{1}=-\frac{\mu_{\odot}}{R^{3}}\left(\boldsymbol{u}_{\odot} \cdot \boldsymbol{r}\right)^{2}$,

also known as tidal acceleration. The cometocentric unit vector $\boldsymbol{u}_{\odot}$ points in the direction of the Sun and $\mu_{\odot}$ is the product of the constant of gravitation and the Sun mass. The comet's heliocentric distance is given by $R$. 


\subsection{Solar radiation}

An important perturber of a spacecraft orbit is radiation pressure. Assuming that incident photons are either absorbed or reflected specularly, neglecting so-called diffuse reflectance (Valorge 1995), an acceleration

$\ddot{\boldsymbol{r}}=-\frac{\xi}{R^{2}}\left[\varepsilon \boldsymbol{u}_{\odot}+2(1-\varepsilon)\left(\boldsymbol{n} \cdot \boldsymbol{u}_{\odot}\right) \boldsymbol{n}\right]$

is induced with the coefficient $\varepsilon \in[0,1]$ and with $\boldsymbol{n}$ as a unit vector normal to the spacecraft surface. In Eq. (5), $\xi$ is a function of the probe's mass, $m_{\mathrm{S} / \mathrm{C}}$, to area factor $\chi_{\odot}$

$\xi=\frac{L_{\odot}}{4 \pi c \chi_{\odot}}, \quad \chi_{\odot}=\frac{m_{\mathrm{S} / \mathrm{C}}}{\left|\boldsymbol{S}_{\mathrm{S} / \mathrm{C}} \cdot \boldsymbol{u}_{\odot}\right|}$.

The solar luminosity is $L_{\odot}, c$ is the velocity of light in vacuum and $\boldsymbol{S}_{\mathrm{S} / \mathrm{C}}=S_{\mathrm{S} / \mathrm{C}} \boldsymbol{n}$ a vector normal to the spacecraft surface with area $S_{\mathrm{S} / \mathrm{C}}$. This vector is defined as a sum over all radiation exposed surface segments of the probe. In this paper we will make the approximations that the magnitude and direction of $\boldsymbol{S}_{\mathrm{S} / \mathrm{C}}$ are entirely determined by Rosetta's large solar cell arrays, unless stated otherwise. This is justified by a total array surface area of $64 \mathrm{~m}^{2}$ and an exposed bus area which is about one tenth of this again. About half of Rosetta's original three tonne mass is fuel, and if all is spent and the solar cell arrays are fully exposed to solar radiation, then $\chi_{\odot}$ obtains its minimum value of about $20 \mathrm{~kg} \mathrm{~m}^{-2}$.

As for gravity, this acceleration is derivable from a potential

$V_{1}=\frac{\xi}{R^{2}}\left[\varepsilon \boldsymbol{u}_{\odot} \cdot \boldsymbol{r}+2(1-\varepsilon)\left(\boldsymbol{n} \cdot \boldsymbol{u}_{\odot}\right) \boldsymbol{n} \cdot \boldsymbol{r}\right]$.

For solar panels $\varepsilon$ is, for obvious reasons, high $\sim 0.8$ (Montenbruck \& Gill 2000). In the case of the Rosetta probe, new technology has been utilized with specially developed nonreflective silicon cells, i.e. $\varepsilon \equiv 1$ for our applications.

\subsection{Outgassing}

\subsubsection{Aerodynamic forces}

If the mean free path of the molecules in the coma is much greater than the size of the probe, i.e. the spacecraft's presence does not perturb significantly the coma dynamics, then we have for the aerodynamic forces acting on the probe (Valorge 1995)

$\ddot{\boldsymbol{r}}=\frac{1}{2} C_{\mathrm{D}} \frac{\varrho(\boldsymbol{r}, t) v(\boldsymbol{r}, t)^{2}}{\chi_{r}}\left(\boldsymbol{u}_{r}+\frac{1}{\eta} \boldsymbol{u}_{\theta}\right), \quad \eta=\frac{C_{\mathrm{D}}}{C_{\mathrm{L}}}$

where $v$ is the adopted radial velocity of the chemical species in question, $\varrho$ is the coma density and $C_{\mathrm{D}}$ and $C_{\mathrm{L}}$ are the drag and lift coefficients respectively. Above $\boldsymbol{u}_{r} \equiv \boldsymbol{r} / r, \boldsymbol{u}_{\theta}$ is a unit vector normal to $\boldsymbol{u}_{r}$ and $\chi_{r}=\chi_{\odot}\left(\boldsymbol{u}_{\odot} \rightarrow \boldsymbol{u}_{r}\right)$.

The radial direction of the drag force is based on the fact that the mostly radially flowing, and collisionally accelerated, gas in certain regions of the coma could have velocities up to about four orders of magnitude, $v_{\mathrm{g}} \sim 1 \mathrm{~km} \mathrm{~s}^{-1}$ (Crifo et al. 1999), larger than the probe velocity in a bound orbit $v_{\mathrm{b}} \sim 0.1 \mathrm{~m} \mathrm{~s}^{-1}$. Shocks deflect flows somewhat (Crifo \& Rodionov 1997a), but can also be described as largely directed away from the nucleus. However, very close to the comet surface and away from the subsolar region, the gas velocity can be almost tangential to a spherical nucleus surface (Crifo et al. 1995), becoming radial first at a few nucleus radii.

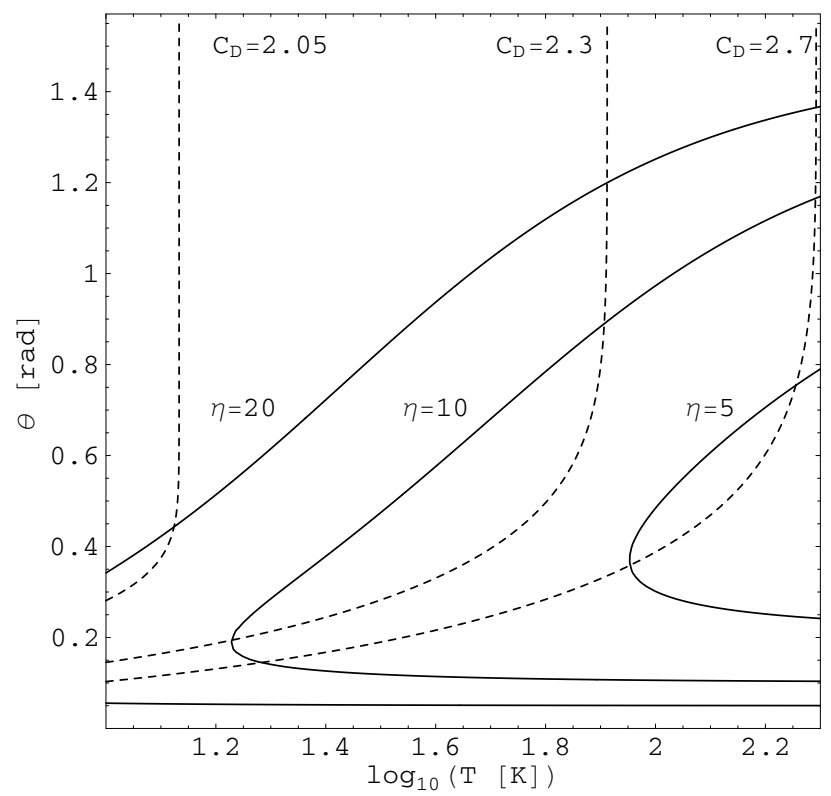

Fig. 1. Level curves for the drag coefficient $C_{\mathrm{D}}$ (- - -) and its ratio to the lift coefficient, $\eta$ (-), as functions of the flow-surface angle $\theta$ (see text), and the coma temperature $T$. The velocity of the flow of water molecules is set to $v=500 \mathrm{~m} \mathrm{~s}^{-1}$ and the arbitrary probe temperature is uniform.

As for the validity of the mean free path of the coma molecules being much larger than the spacecraft, this is, among many things, dependent on the total production rate of the different species and the cometocentric angle between the probe and the Sun. Adopting a cross section $\sigma \sim 3 \times 10^{-18} \mathrm{~m}^{2}$ for the coma molecules (Rodionov et al. 2002), a simple expression for the mean free path, $\Delta x=\left(\sqrt{2} \sigma \varrho_{n}\right)^{-1}$, yields a coma number density $\varrho_{n} \lesssim 1 \times 10^{15} \mathrm{~m}^{-3}$ for the approximation to be fair. This value is similar to the density at $r=6-7 r_{\mathrm{c}}$ (Crifo et al. 2004) in a worstcase CO production scenario (Bockelée-Morvan et al. 2004). However, the perihelion water production rate of comet $67 \mathrm{P}$ is an order of magnitude larger than this again (see later), and the molecules are most certainly less uniformly distributed in the coma than for the more volatile $\mathrm{CO}$. Only further away from the nucleus will the approximation Eq. (8) then hold.

\subsubsection{Drag and lift}

A common assumption for the calculation of $C_{\mathrm{D}}$ and $C_{\mathrm{L}}$ is that the incident molecules' velocities are thermalized by the walls of the spacecraft before they are re-emitted. Such socalled high accomodation by the exposed surfaces are based on both orbital studies and experiments, consistent with for instance the development of a gaseous layer on the probe surface (King-Hele 1987) or massive gas molecules (Valorge 1995) like $\mathrm{CO}$ or $\mathrm{H}_{2} \mathrm{O}$. As a result, $C_{\mathrm{D}}$ and $\eta$ are not only dependent on the angle $\theta \in[0, \pi / 2]$ between $\boldsymbol{u}_{r}$ and its component in the plane normal to $S_{\mathrm{S} / \mathrm{C}}$, as well as the ratio $s=v T^{-1 / 2}$ where $T$ is the coma temperature, but also on the difference between the temperatures on the front, $T_{1}$, and on the rear side, $T_{2}$, of a flat plate (Valorge 1995). In the following, it will also be assumed that the molecule re-emission is diffuse with preference along the surface normal, which could be representable for low temperature surfaces (Cook 1965). Shown in Fig. 1 are the level curves of $\eta$ and $C_{\mathrm{D}}$ in the $(T, \theta)$ plane for any $T_{1}=T_{2}$. Figure 2 illustrates the results for $T_{1}=300 \mathrm{~K}$ and $T_{2}=0 \mathrm{~K}$. 


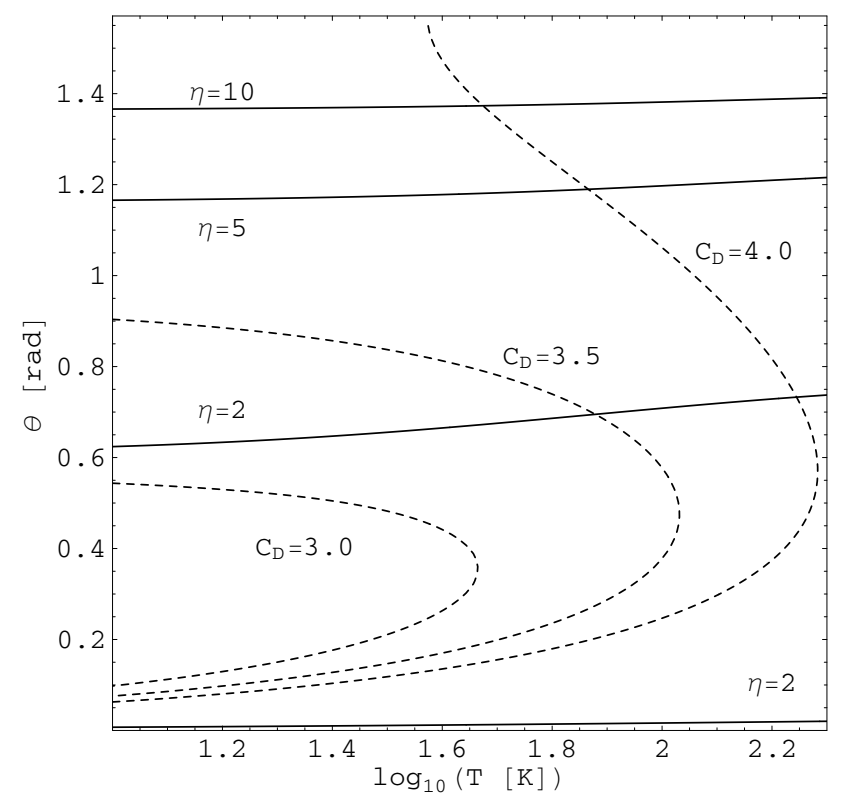

Fig. 2. Contours for the drag coefficient $C_{\mathrm{D}}(---)$ and $\eta(-)$ as functions of the flow-surface angle $\theta$, and the coma temperature $T$. The water flow velocity is set to $v=500 \mathrm{~m} \mathrm{~s}^{-1}$, and the values $T_{1}=300 \mathrm{~K}$ and $T_{2}=0 \mathrm{~K}$ are used.

In for instance Crifo (1987) and Crifo et al. (2003) one can clearly see how the coma temperature decreases rapidly away from the nucleus down to between 10 and $100 \mathrm{~K}$ due to expansion cooling. On the basis of Figs. 1 and 2 we will therefore in this paper not study the $C_{\mathrm{L}}$ effects, an approximation which defines the lowest possible $\theta$ as a function of coma and spacecraft temperatures. The drag coefficient $C_{\mathrm{D}}$ will for convenience be set to a minimum of two in the rest of this paper, yielding a possible underestimation of the drag force by the same factor, Fig. 2. On the other hand, this correction is reset to zero at the early stages of the mission due to our later assumption that all of Rosetta's fuel has been spent. That is, the mass of Rosetta at the beginning of the mission is about twice of what we assume throughout this paper.

We do not exclude, except as simplifying approximations in this paper, the possibilities of low accomodation and that the molecules may remember the direction of their pre-impact velocities (specular reflection).

\subsubsection{Radial dependency}

For a stationary flow, mass is conserved in a spherical volume outside the comet nucleus

$m Q=r^{2} \oint \mathrm{d} \Omega \varrho v, \quad \varrho v \sim \mathcal{F}(r, \lambda, \phi)$

where the integration is over the outer sphere. The chemical species' production rate is represented by $Q\left(\mathrm{~mol} . \mathrm{s}^{-1}\right), m$ is its molecular weight and $\mathrm{d} \Omega=\mathrm{d} \lambda \mathrm{d} \phi \sin \phi$ a cometocentric solid angle. Here $\lambda$ is an angle in the plane normal to $\boldsymbol{u}_{\odot}$ and $\cos \phi=\boldsymbol{u}_{\odot} \cdot \boldsymbol{u}_{r}$. Substituting Eq. (9) back into Eq. (8) yields

$\ddot{\boldsymbol{r}}=\frac{1}{\chi_{r}} \frac{m Q}{\oint \mathrm{d} \Omega^{\prime} \mathcal{F}\left(r, \lambda^{\prime}, \phi^{\prime}\right)} \frac{\mathcal{F}(r, \lambda, \phi)}{r^{2}} v(\boldsymbol{r}) \boldsymbol{u}_{r}$.

Note that if the mass flux is separable, $\mathcal{F}=\mathcal{F}_{r}(r) \mathcal{F}_{\Omega}(\lambda, \phi)$, and the velocity stabilizes $v \neq v(r)$ at some distance away from the nucleus due to the inevitable lower coma density further out, the acceleration Eq. (10) goes as $\sim r^{-2}$. This is the same dependency as that for a free outflow of molecules, which may never be the case, even at the comet's aphelion (Crifo \& Rodionov 1997a). More specifically, calculations for the old Rosetta target 46P/Wirtanen showed that free outflow conditions will not be encountered in its dayside coma at heliocentric distances $R<3 \mathrm{AU}$ (Crifo \& Rodionov 1997a), after the onset of water sublimation. Beyond this distance, the occurrence of free outflow is dependent on the absence of chemical species which are more volatile than water, and therefore less modulated by heliocentric distance (Capria et al. 2004). Although some recent observations did not detect the presence of the volatile CO (Bockelée-Morvan et al. 2004), the existence of such reactive species can be inferred on the basis of a large dust mass loss rate at a distance of low or negligible water production (Fulle et al. 2004), where the dust grains must have been lifted from the nucleus by the production of some other type of species.

\subsection{Dust drag}

According to Fulle et al. (2004), Rosetta will encounter a dust mass loss rate of $100 \mathrm{~kg} \mathrm{~s}^{-1}$, dominated by $\mathrm{cm}$-sized grains, during its approach to 67P. Although this rate is, as we shall see, in mass comparable to the worst-case $\mathrm{CO}$ production, dust will here not be assumed to influence the dynamics of the orbiter due to the grains' low velocity $v_{\mathrm{d}} \sim 1 \mathrm{~m} \mathrm{~s}^{-1}$ (Crifo et al. 2004), see Eq. (8).

\subsection{Recoil force on nucleus}

The outgassing from the comet nucleus results in a recoil force which can be estimated from astrometric data. In Królikowska (2003) the associated acceleration is represented by a product of an estimated strength $A \sim 10^{-9} \mathrm{AU} \mathrm{d} \mathrm{d}^{-2}$ and a function $g(R)$ which is based on the sublimation physics of water. In the accelerated reference frame of the comet, this induces a cometocentric acceleration of the spacecraft with opposite direction to that of the force on the nucleus. The force on the nucleus acts mainly in the anti-solar direction.

\section{Comparison of accelerations}

\subsection{Assumptions}

The different accelerations Eqs. (3), (5), (4) and (8) must be compared so that we can anticipate the feasibility of some of the Rosetta mission's different goals. That is, the possibilities for inversion of the detailed gravity field and bound orbits. All accelerations will here be computed with the simplest possible techniques, just so that a comparison can be made. Therefore, there are aspects of motion and relative strengths which are neglected in this preliminary analysis, like when the contribution from an effect vanishes entirely due to a particular choice of orbit or solar cell array geometry.

None of the cometary flybys before NASA's Deep Impact perturbed the probes enough to yield estimates of the nucleus masses (Anderson et al. 2004). However, 67P's mass has been given a solid upper limit of $m_{\mathrm{c}} \leq 2.1 \times 10^{13} \mathrm{~kg}$ (Davidsson \& Gutiérrez 2005) by comparing the nucleus' water production rates and observed non-gravitational displacements of the comet's orbit. As pointed out in their work, Rickman et al. (1987) had previously constrained the mass 
of $67 \mathrm{P}$ to $0.1<m_{\mathrm{c}}<1.3 \times 10^{13} \mathrm{~kg}$, and we will therefore, based on these studies, use

$m_{\mathrm{c}}=0.1$ (low) -1 (nominal) $\times 10^{13} \mathrm{~kg}$.

For the purpose of comparing the different effects the normalized (Heiskanen \& Moritz 1967) coefficient $\bar{c}_{20} \equiv 0.1$, and can be adopted as definition of the reference radius $r_{\mathrm{c}}$ which here is set to $r_{\mathrm{c}}=1.98 \mathrm{~km}$ (Lamy et al. 2003). Furthermore, both the pressure and the tidal accelerations are maximized by an unfavourable geometry which for the radiation pressure Eq. (5) corresponds to the angle between the solar cell arrays $S_{\mathrm{S} / \mathrm{C}}$ and the Sun direction $\boldsymbol{u}_{\odot}$ being zero. The resulting mass to exposed area factor is likewise set to a minimum $\chi_{r, \odot}=20 \mathrm{~kg} \mathrm{~m}^{-2}$.

For the continuous outgassing, a simplified mass flux function, Eq. (9),

$\mathcal{F}(\phi)=\sum_{i=0}^{n} \tilde{\alpha}_{i} \cos ^{i} \phi, \quad v \neq v(\boldsymbol{r})$

where $\tilde{\alpha}_{i}$ are coefficients to be determined, is often adopted, corresponding to the case of a spherical nucleus with no heat conduction properties and a very symmetric activity pattern. That is, there is no direction of preference other than that of the Sun. However, according to Crifo \& Rodionov (1997b), the circumnuclear coma structure is by no means (in general, authors' comments) axially symmetric around the comet-to-Sun axis. The probe's cometocentric acceleration following Eq. (12) is not derivable from a potential, as can be verified from $\nabla \times \ddot{\boldsymbol{r}} \neq \mathbf{0}$ with $\ddot{r}$ of Eq. (10) for $i \neq 0$.

In order to evaluate the strength of the continuous pressure field of the outflowing water molecules, the day side emphasized profile as a subgroup of Eq. (12)

$\mathcal{F}(\phi) \sim \cos \phi \Theta_{\mathrm{S}}\left[\cos \phi-\cos \phi_{\mathrm{c}}\right], \quad \phi_{\mathrm{c}} \leq \pi / 2$,

is adopted where $\Theta_{S}$ is the step function, meaning that the pressure field goes as $\cos \phi$ until the cut-off angle $\phi_{\mathrm{c}}$. The normalization is

$\frac{\mathcal{F}(\phi)}{\oint \mathrm{d} \Omega^{\prime} \mathcal{F}\left(\phi^{\prime}\right)}=\frac{2 \cos \phi}{\pi\left(1-\cos 2 \phi_{\mathrm{c}}\right)} \Theta_{\mathrm{S}}\left[\cos \phi-\cos \phi_{\mathrm{c}}\right]$

yielding an enhancement, relative to a spherical symmetric pressure field, of 8 at $\phi=0$ for $\phi_{c}=\pi / 4$. The physicality of this maximum, even in the presence of shocks, will be brought up later in this paper. In addition the high

$v_{\mathrm{H}_{2} \mathrm{O}} \sim 1 \mathrm{~km} \mathrm{~s}^{-1}$

is used in the following comparison. Order of magnitude modifications of this pressure field are easy to take into account from the coming plots.

The water production rate (Teemu \& Mäkinen 2004)

$Q_{\mathrm{H}_{2} \mathrm{O}} \sim 1 \times 10^{28}$ mol. $\mathrm{s}^{-1}$

at perihelion $R=1.3 \mathrm{AU}$ is adopted, while the exponential fit of Oertzen (2003) yields

$Q_{\mathrm{H}_{2} \mathrm{O}}(R=2.5 \mathrm{AU}) \sim 1 \times 10^{26} \mathrm{~mol} . \mathrm{s}^{-1}$

and

$Q_{\mathrm{H}_{2} \mathrm{O}}(R=3.5 \mathrm{AU}) \sim 5 \times 10^{23} \mathrm{~mol} . \mathrm{s}^{-1}$.

Water outgassing is generally considered to be negligible beyond 3-4 AU (Crifo et al. 1999).

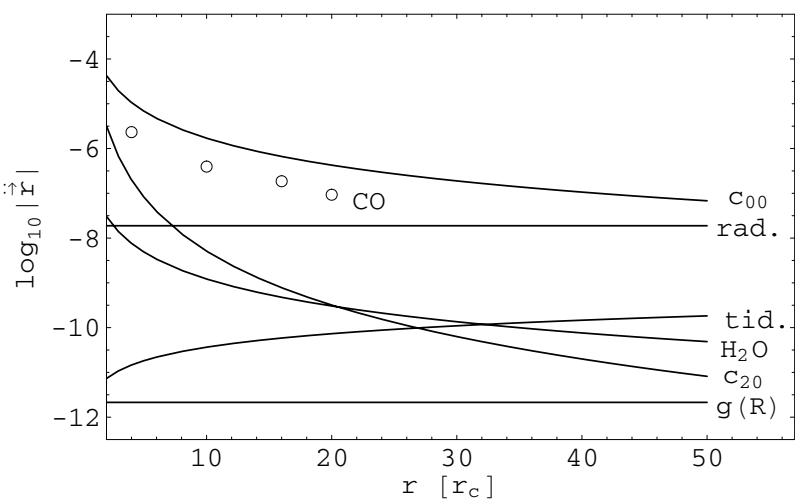

Fig. 3. Cometocentric acceleration $\log _{10}\left|\ddot{\boldsymbol{r}}\left[\mathrm{m} \mathrm{s}^{-2}\right]\right|$ of a nearby probe for a nominal nucleus mass at heliocentric distance $R=3.5 \mathrm{AU}$. The spherical symmetric gravitational attraction is represented by $c_{00}$, while the meaning of the other curves should be evident from the context.

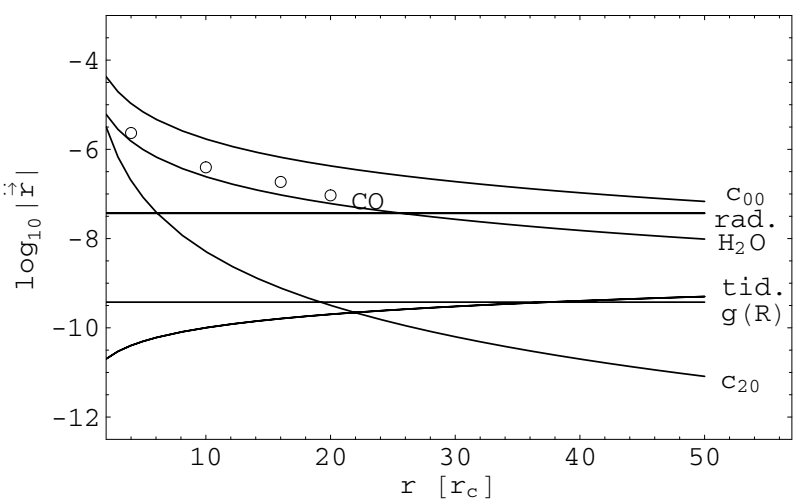

Fig. 4. Acceleration of a nearby probe relative to a nominal nucleus mass at heliocentric distance $R=2.5 \mathrm{AU}$.

The CO drag factors $\varrho v^{2}$ are extrapolated from the 67P coma simulations of Crifo et al. (2004) with near uniform CO outgassing, and high global production rate of Bockelée-Morvan et al. (2004)

$Q_{\mathrm{CO}} \sim 1 \times 10^{27}$ mol. $\mathrm{s}^{-1}$.

Taking into account Capria et al. (2004)'s simulations showing a weaker production dependency on $R$ for this volatile, the rate above will be assumed to represent the species' drag at all heliocentric distances. However, the nature of $\mathrm{CO}$ production for comet 29P/Schwassmann-Wachmann 1 (Gunnarsson et al. 2002) indicates a dependency on heliocentric distance as was observed for long-period comet Hale-Bopp (Biver et al. 1997). On the other hand, Hale-Bopp and Centaur 29P have different thermal histories than 67P (De Sanctis et al. 2005). The strength of this extrapolated $\mathrm{CO}$ pressure field is seen to drop roughly as $\varrho v^{2} \sim r^{-2}$.

\subsection{Discussion}

Maximizing our adopted profile Eq. (14) with $\phi_{\mathrm{c}}=\pi / 4$ and $\phi=0$, we obtain the acceleration results of Figs. 3-5, representing different heliocentric distances and the nominal nucleus mass. The open circles represent the sampled $\mathrm{CO}$ values along the comet-Sun line. It is easy to judge the effect of an order of magnitude mass reduction from the plots.

From the figures it can be seen that worst-case $\mathrm{CO}$ outgassing could compromise the existence of stable bound orbits in general at any heliocentric distance if the nucleus mass is 


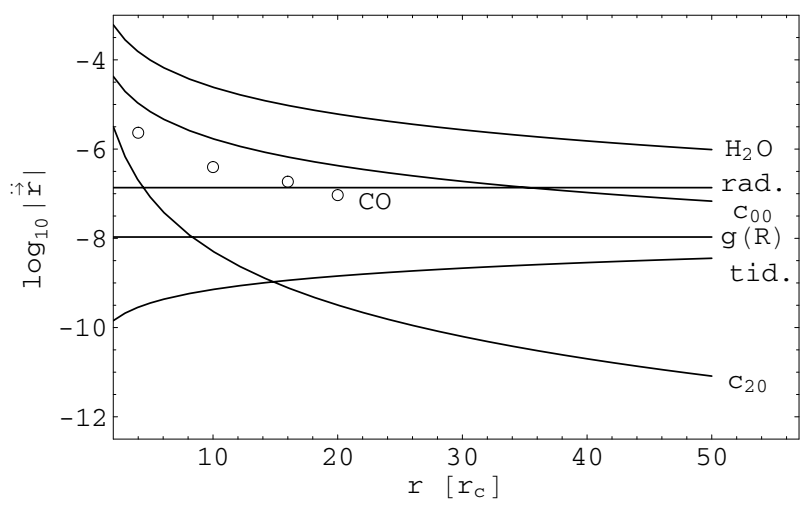

Fig. 5. Accelerations of a spacecraft orbiting a nominal nucleus mass at heliocentric distance $R=1.3 \mathrm{AU}$.

less than nominal. In this paper however, we define the nominal rate to be an order of magnitude lower than the inferred upper limit Eq. (19), but this may be an uncertain assumption. At this stage, it is interesting to point out that the $\mathrm{CO}$ production rate of (19) was below the detection limit a few years back (Bockelée-Morvan cited in Enzian et al. 1999). The effect of outgassing can be reduced another order of magnitude by not exposing the orbiting probe's solar cell arrays, i.e. make $\left|\boldsymbol{S}_{\mathrm{S} / \mathrm{C}} \cdot \boldsymbol{u}_{r}\right|$ small, but the spacecraft bus will always project an area in the comet nucleus' direction. The possible rotation of the probe's arrays relative to the Sun could, on the other hand, be dynamically hazardous. Resonances between the rotation period(s) of the probe and its orbit could be strong enough to make the trajectory chaotic through the spacecraft's interaction with solar radiation, a phenomenon which is beyond the scope of this paper.

Notice the non-sphericity component of the gravity field, the $c_{20}$ curve, to be detected and inverted as part of the Rosetta RSI (Pätzold et al. 2001). If the CO pressure field of Eq. (19) is down-scaled an order of magnitude for it to represent the defined nominal rate, the corresponding acceleration is comparable to the rapidly dropping non-sphericity component of the gravity field at $r \sim 10 r_{\mathrm{c}}$, as long as the exposed area is minimized.

Except for the pressure from the more volatile chemical species, radiation pressure is the most important perturber of Keplerian motion at $R=3.5 \mathrm{AU}$, surpassed by higher degree gravity close to the comet nucleus. At the same comet heliocentric distance, radiation pressure becomes comparable to the comet's gravitational attraction for trajectories above $r \sim 40 r_{\mathrm{c}}$, and the existence of bound orbits can certainly be questioned. Also, the prospect of Rosetta orbiting the comet through its perihelion should be investigated more closely since the pressure from the outflowing water molecules overcomes the gravitational attraction from the nucleus. As suggested by Scheeres \& Marzari (2002), the spacecraft can then be placed in a generalized Lagrangian point between the Sun and the comet which, due to the strong radiation pressure perturbation, is displaced towards the Sun at a safer distance from the comet. Moreover, radiation pressure significantly weakens the instability of this point.

\section{First-order perturbation theory}

We have seen that bound cometocentric orbits seem plausible for nominal parameters at $R=3.5 \mathrm{AU}$, and that this motion preliminarily can be approximated as Keplerian. Perturbations like outgassing and radiation pressure alter this picture by changing the geometry of the orbit as the probe traverses it. The nature of these changes can be fundamentally different for the various perturbers. For instance, while the acceleration connected to the higher harmonics Eq. (3) obviously still is small at $r \lesssim 5 r_{\mathrm{c}}$, it could rapidly change the orbital energy (Mysen \& Aksnes 2005). These so-called chaotic changes, generic for Hamiltonian systems, are very much present in our case study, and pose exceptional challenges for the Rosetta mission since two initially nearby trajectories diverge exponentially with time. That is, predictions become more difficult as a result of the uncertainty in boundary and initial conditions.

However, we will focus mostly on the dynamical regions where the perturbation still can be considered weak, i.e. where the dynamics is regular and non-resonant. Assume then that we have a set of differential equations for variables $x$ which here are defined to be constant for two-body motion

$\dot{x}=f_{\Sigma}(x, t)$

where the right-hand side is a small quantity, indicating that we are dealing with a perturbation. Usually, $f_{\Sigma}$ is periodic in some system angles that change in zero-order motion, like the orbiter's cometocentric mean anomaly $\tilde{l}$. The right hand side of Eq. (20) can then be Fourier expanded

$\dot{x}=f_{\mathrm{s}}(x)+f_{\delta}(x, \tilde{l})$

where

$f_{\mathrm{s}}=\frac{1}{2 \pi} \int_{0}^{2 \pi} \mathrm{d} \tilde{l} f_{\Sigma}, \quad f_{\delta}(\tilde{l})=f_{\delta}(\tilde{l}+2 \pi)$.

Now, the basis for a rigorous (Hori 1966) first-order perturbation theory is that an approximation to the motion can be obtained iteratively, i.e. by substituting the unperturbed system into the right hand-side of Eq. (21), and then integrating. The Fourier expansion should then have been made in the angles which vary linearly in time in zero-order motion so that the time integration of $f_{\delta}$ then results in a strictly periodic component. For limited time-spans the system therefore evolves according to

$x(t)=\bar{x}(t)+\delta x\left[\bar{x}\left(t_{0}\right), \tilde{l}\right], \quad \delta x(\tilde{l})=\delta x(\tilde{l}+2 \pi)$

where the mean state is represented by

$\bar{x}(t)=\bar{x}\left(t_{0}\right)+f_{\mathrm{s}}\left[\bar{x}\left(t_{0}\right)\right]\left(t-t_{0}\right)$,

also called secular since its change usually is slow compared to the zero-order frequencies. Naturally, within the time-scale of the linear approximation above, the contribution from each perturbing effect to the change in $x$ can be treated separately. For longer periods of time however, the coupled equation

$\dot{\bar{x}}=f_{\mathrm{s}}(\bar{x})$

must be solved, yielding a solution with error less than $\sim f_{\Sigma}$ on a time-scale less than $\sim f_{\Sigma}^{-1}$. The right hand side above is then the sum of the angle-independent part $f_{\mathrm{s}}$ of all perturbers.

If $f_{\Sigma}$ is periodically dependent on yet another angle $\theta$ which changes rapidly, the averaging process Eq. (22) must be continued until all fast "zero-order" angles are removed, i.e.

$f_{\mathrm{s}}=\frac{1}{2 \pi} \int_{0}^{2 \pi} \mathrm{d} \theta \frac{1}{2 \pi} \int_{0}^{2 \pi} \mathrm{d} \tilde{l} f_{\Sigma} \equiv \bar{f}_{\Sigma}, \quad$ etc.

These can for instance be the rotational phases of the nucleus and solar cell arrays. Interestingly, if the most rapidly changing angles are removed first, the incomplete angle average has a clear physical content. That is, motion derived from the incomplete angle mean deviates from the true state of the system only by 
the smallest and most rapidly oscillating part of the periodic $\delta x$, Eq. (23). We shall return to this aspect later on.

For a system which is derivable from a potential $V_{1}$, the time derivatives of some otherwise constant state parameters can be found by taking the partials of this function with respect to certain dynamical variables. This greatly simplifies the averaging procedure since only one function needs to be averaged. The Delaunay impulse (Kovalevsky 1967) directly related to the cometocentric semi-major axis $a$ of the orbiting probe, obeys

$\dot{\bar{L}}=-\frac{\partial \bar{V}_{1}}{\partial \tilde{l}}=0, \quad L=\sqrt{\mu_{\mathrm{c}} a}$,

and the Keplerian energy $F_{0}=-\mu_{\mathrm{c}} / 2 a$ is therefore conserved on average, a well-known property of periodic systems in the regular dynamical regime. Likewise, the derivatives of the orbit angular momentum

$\dot{\bar{G}}=-\frac{\partial \bar{V}_{1}}{\partial \bar{g}}, \quad G=L \sqrt{1-e^{2}}$,

and its component along some reference $Z$-axis

$\dot{\bar{H}}=-\frac{\partial \bar{V}_{1}}{\partial \bar{h}}, \quad H=G \cos I$,

can easily be worked out. $g$ and $h$ above are the orbit's argument of pericenter and longitude of ascending node from a reference $X$-axis, respectively, while $e$ is the orbit eccentricity and $I$ its inclination with respect to some reference $X Y$ plane. In what follows, the special notation introduced to indicate that we are dealing with averaged or mean quantities is neglected for the dynamical variables themselves.

\section{Gravitational perturbations}

Regarding the physical content of only partially averaged differential equations, Mysen (2005) has shown explicitly that for extended periods of time, and if the orbit is much larger than the synchronous radius $r_{\mathrm{s}}$, the nucleus' rotational phases can be removed from Eq. (2) by a procedure like Eq. (26) without compromising the resulting model, $\hat{V}_{1}$, as a representative description of the spacecraft's gravitational acceleration. According to Mysen (2004)

$\hat{V}_{1}=\frac{\mu_{\mathrm{c}}}{r} \sum_{n=2}^{\infty} c_{n 0} \hat{\psi}_{n}\left(\frac{r_{\mathrm{c}}}{r}\right)^{n} P_{n}(\sin \vartheta)$

where $\vartheta$ is the cometocentric declination of the spacecraft relative to the plane normal to the nucleus' rotational spin $\boldsymbol{G}_{\mathrm{c}}$ and $P_{n}$ are the Legendre polynomials. The $\hat{\psi}_{n}$ factors are dependent on the mass distribution and rotational excitation of the comet nucleus, and has previously been derived by Kinoshita (1972) for the case $n=2$, although under a different name and in a different order than in the reference above. The $\hat{\psi}_{2}$ excitation factor has also been given by Scheeres et al. (1998b) for the limiting case of a rotationally symmetric oblate body

$\hat{\psi}_{2}=\frac{1}{2}\left(3 \cos ^{2} J_{\mathrm{c}}-1\right)$,

where $J_{\mathrm{c}}$ is the, then, constant angle between the nucleus equator and its spin plane. For the reported rotation period $P_{\mathrm{c}}=12.3 \mathrm{~h}$ of Lamy et al. (2003) and a nominal nucleus mass, we have $r_{\mathrm{s}}=$ $1.6 r_{\mathrm{c}}$.
The transition to the completely averaged $\bar{V}_{1}$ can easily (Roy 1998) be effected by the mean anomaly operation Eq. (22) on $\hat{V}_{1}$. Neglecting the higher order harmonics

$\bar{V}_{1}=-\frac{\mu_{\mathrm{c}}}{a} c_{20} \hat{\psi}_{2}\left(\frac{r_{\mathrm{c}}}{a}\right)^{2} \frac{\hat{Q}}{8\left(1-e^{2}\right)^{3 / 2}}$,

valid for arbitrary spin plane inclinations $I_{\mathrm{c}}$ with respect to the reference $X Y$ plane, where (Kinoshita 1972)

$$
\begin{aligned}
\hat{Q}=( & \left.3 \cos ^{2} I-1\right)\left(3 \cos ^{2} I_{\mathrm{c}}-1\right) \\
& +3 \sin 2 I \sin 2 I_{\mathrm{c}} \cos \Delta h_{\mathrm{c}}+3 \sin ^{2} I \sin ^{2} I_{\mathrm{c}} \cos 2 \Delta h_{\mathrm{c}} .
\end{aligned}
$$

with $\Delta h_{\mathrm{c}}=h_{\mathrm{c}}-h$ as the difference, in the reference plane, between the node of the plane normal to the nucleus spin, and the orbit's longitude of ascending node.

The semi-major axis of the orbit is according to Eq. (27) secularly conserved under the action of gravitation alone. Interestingly, $g$ is not contained in $\hat{Q}$ either. Together with the conservation of energy, this means that the mean eccentricity is very nearly conserved, but only nearly since the averaged third harmonics, $n=3$, can be shown to contain $g$, Eq. (28). If $I_{\mathrm{c}} \equiv 0$, i.e. the reference plane coincides with the plane normal to the nucleus spin, the node $h$ is removed from all harmonics already at the stage Eq. (30). That is, the component of the orbital angular momentum $\boldsymbol{G}$ on the spin axis of the nucleus is secularly constant. Also, the classic expressions for node and pericenter precession are rediscovered for $I_{\mathrm{c}} \equiv 0$, but with the modifying excitation factor $\hat{\psi}_{2}$. For instance

$\dot{g}=\frac{\partial \bar{V}_{1}}{\partial G}=\frac{3}{4} n \frac{c_{20} \hat{\psi}_{2}}{\left(1-e^{2}\right)^{2}}\left(\frac{r_{\mathrm{c}}}{a}\right)^{2}\left(5 \cos ^{2} I-1\right)$

where $n^{2} a^{3}=\mu_{\mathrm{c}}$.

For $67 \mathrm{P}$, the time spent by the comet from $4 \mathrm{AU}$ to its perihelion is about $10^{4} \mathrm{~h}$. During this period $\Delta g \lesssim 1$ at $a=10 r_{\mathrm{c}}$ from Eq. (34). That is, secular gravitational effects will be neglected in our applications.

\section{The solvable radiation pressure problem}

\subsection{Secular integrals}

Averaging the potential Eq. (7) with full photon absorption by the solar cell arrays results in

$\bar{V}_{1}=\frac{\bar{\xi}}{R^{2}} \frac{1}{2 \pi} \int_{0}^{2 \pi} \mathrm{d} \tilde{l} \boldsymbol{u}_{\odot} \cdot \boldsymbol{r}=-\frac{3}{2} \frac{\bar{\xi}}{R^{2}}$ a e $\boldsymbol{u}_{\odot} \cdot \boldsymbol{u}_{\mathrm{p}}$,

where the bar indicates that all zero-order angles have been removed, as in Eq. (26), and $\boldsymbol{u}_{\mathrm{p}}$ is a cometocentric unit vector in the direction of the orbiting probe's pericenter. Inserting the average Eq. (35) into Lagrange's equations (Kovalevsky 1967), as is commonly done for this problem, yields the time derivatives of the mean non-canonical cometocentric orbital elements $a, e$, $I, h$ and $g$ of the orbiting probe, where $\dot{a}=0$ consistent with previous considerations. The comet comoving reference system used for the calculation of the angles, and later simulations, is shown in Fig. 6.

However, it is more convenient to formulate the change in the orbital elements in terms of the comet nucleus' true anomaly $v$, yielding a perturbation with a dimensionless strength independent of the heliocentric distance for the non-canonical variables (Scheeres 1999)

$\Lambda=\frac{3}{2} \frac{\bar{\xi}}{\sqrt{\mu_{c} \mu_{\odot} A_{\mathrm{C}}\left(1-E_{\mathrm{C}}^{2}\right)}} \sqrt{a}=\left\{\begin{array}{l}2.06 \\ 6.52\end{array}\right\} \sqrt{\frac{a}{r_{\mathrm{c}}}}$. 


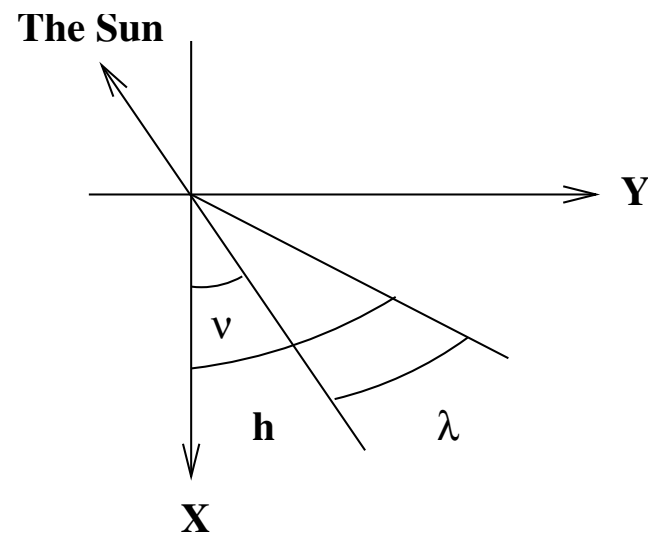

Fig. 6. A commonly adopted reference system for the radiation pressure problem. The comet nucleus' heliocentric true anomaly $v$ and $\lambda=h-v$, the difference between the orbiter's longitude of ascending node and this true anomaly, are shown.

Here $A_{\mathrm{C}}$ and $E_{\mathrm{C}}$ are the semi-major axis and eccentricity of the comet in its orbit around the Sun, and the two values correspond to the low and nominal nucleus mass, both evaluated at a minimized mass to area factor. Note that the cometary orbiter's semimajor axis has been included in the strength itself since it is a secular constant.

The averaged equations of motion have been solved by Mignard \& Henon (1984), uncovering several integrals on their way. Two interesting and important properties of the radiation pressure induced secular motion are, as a result, easily available for the case $I_{0} \neq 0$. For $I_{0}=0$ the reader is referred to Mignard (1982). First of all $\sin ^{2} I\left(1-e^{2} \cos ^{2} g\right)$ is secularly conserved, with the implication that the inclination can never become smaller than a certain value $I_{m}$ (Scheeres 1999)

$\sin ^{2} I \geqq \sin ^{2} I_{m}=\sin ^{2} I_{0}\left(1-e_{0}^{2} \cos ^{2} g_{0}\right)$.

Secondly, a quantity $-\mathcal{X} \Lambda+\mathcal{Z}$ is also secularly conserved. Since both $\mathcal{X}$ and $\mathcal{Z} \in[-1,1]$, and $\Lambda$ is large, we will approach the qualitative aspects of the motion by adopting $-\mathcal{X}=$ $\sqrt{1-e^{2}} \sin I \sin \lambda$ as a secular constant

$1-e^{2}=\frac{X_{0}^{2}}{\sin ^{2} I \sin ^{2} \lambda}$

where $\lambda$ here is defined by $\lambda=h-v$. First of all, Eq. (38) implies that the orbital plane is limited to exist in the vicinity of the socalled solar plane-of-sky, where the denominator of the equation by definition equals one.

More interestingly, the eccentricity reaches its maximum value for this denominator, a situation which is encountered in full simulations of the equations of motion. However, when the maximum eccentricity $e_{\mathrm{M}}$ derived in this way is small, the neglectance of the term $\mathcal{Z} / \Lambda \sim e / \Lambda$ in the conservation law above could alter the result significantly. A more elaborate calculation yields

$$
\begin{aligned}
\mathcal{X}^{2}-\mathcal{X}_{0}^{2} \approx 2 \Lambda^{-1}\left(\mathcal{X}-\mathcal{X}_{0} \mathcal{Z}_{0}\right) \\
>-2 \Lambda^{-1}\left(e_{M}+\sin \gamma_{0}\right)
\end{aligned}
$$

where

$\sin \gamma_{0} \equiv e_{0} \sqrt{1-e_{0}^{2}} \sin ^{2} I_{0} \sin \lambda_{0} \sin g_{0}$,

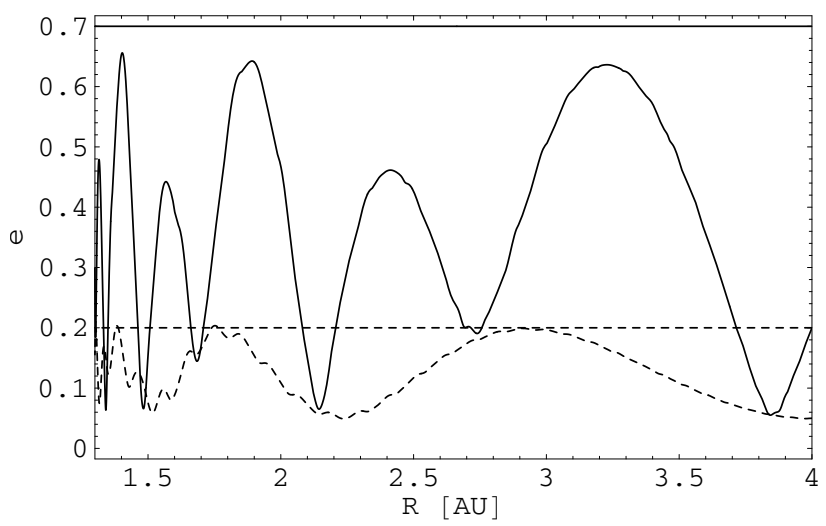

Fig. 7. The radiation pressure induced variation of an orbiting probe's eccentricity as a function of the comet nucleus' heliocentric distance before perihelion.

from which we obtain the minimum $X^{2}$. Making the substitution $\mathcal{X}^{2} \rightarrow 1-e_{\mathrm{M}}^{2}$, the maximum of the mean eccentricity can be found

$e<e_{\mathrm{M}}=\Lambda^{-1}+\sqrt{\Lambda^{-2}+1-\mathcal{X}_{0}^{2}+2 \Lambda^{-1} \sin \gamma_{0}}$.

As a demonstration, a simulation of Eq. (5) using a nominal nucleus mass with (solid)

$e_{0}=0.2, \quad a_{0}=15 r_{\mathrm{c}}, \quad I_{0}=\lambda_{0}=g_{0}=1.2$

where $e_{\mathrm{M}}=0.70$, and another one with (dashed)

$e_{0}=0.05, \quad a_{0}=15 r_{\mathrm{c}}, \quad I_{0}=-\lambda_{0}=g_{0}=\pi / 2$,

having an $e_{\mathrm{M}}=0.20$, are shown in Fig. 7. From the details behind inequality (39), the predicted secular maximum is most conservative for high $e_{\mathrm{M}}$ 's, consistent with the simulations. Also, notice the approximate periodicity which can be traced back to the fact that the solved equations for the secular problem are periodic in an angle $\mathcal{H}$ (Mignard \& Henon 1984) where

$\mathcal{H}=\frac{v-v_{0}}{\cos \psi}, \quad \tan \psi=\Lambda$.

So, if the solar cell arrays' exposure to radiation is minimized, i.e. relatively low $\Lambda$ of Eq. (36), the secular change in eccentricity is correspondingly slow.

From Eq. (41), the maximum eccentricity is minimized by choosing the orbit to lie in the solar plane-of-sky where $\mathcal{X}_{0}^{2}$ is large, with for instance $I_{0}=\lambda_{0}=\pi / 2$, avoiding close pericenter passages where chaotic gravitational perturbations prevail (Mysen \& Aksnes 2005), and distant apocenters where the regularity of the radiation pressure induced motion can be questioned. From the same equation, a further reduction in $e_{\mathrm{M}}$ can be achieved with a proper choice of pericenter

$\sin \lambda_{0} \sin g_{0}=-1 \Longrightarrow \sin \gamma_{0}<0$.

It is important to note that this configuration gives the minimum of all $e_{\mathrm{M}}$ 's, and that this value is not obtained by $e_{0}=0$, but by $e_{0} \approx \Lambda^{-1}$, see the square root of Eq. (41).

\subsection{The solar plane-of-sky equilibria}

In the limit of strong radiation pressure perturbation, as is the case with our large $\Lambda$ 's, this configuration coincides with Scheeres et al. (1998a)'s secular equilibria (in mean variables)

$\lambda^{*}= \pm \pi / 2, \quad g^{*}=\mp \pi / 2, \quad I^{*}=\pi / 2, \quad e^{*}=\cos \psi$ 


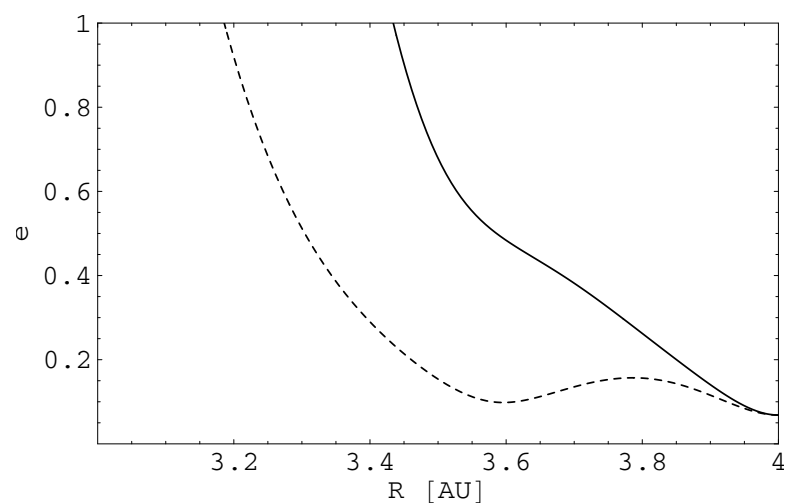

Fig. 8. Evolution of the eccentricity for the probe in an initially bound orbit around a nominal 67P nucleus, with instantaneous initial conditions equal to those of the equilibrium (dashed), or those of the mean equilibrium (solid).

where $\psi$ is given by Eq. (44), see Fig. 6. If

$\frac{\mathrm{d} x}{\mathrm{~d} v}=f_{x}(x), \quad x=(\lambda, I, g, e)$,

we have by definition the linearized motion $\delta x=x-x^{*}$

$\frac{\mathrm{d}}{\mathrm{d} v} \delta x=f_{x}\left(x^{*}\right)+\frac{\partial f_{x}}{\partial x^{*}} \delta x, \quad f_{x}\left(x^{*}\right) \equiv 0$,

where two large elements of the stability matrix are (Scheeres 1999)

$\frac{\partial}{\partial I^{*}} \frac{\mathrm{d} g}{\mathrm{~d} \nu}=\frac{1}{\cos ^{2} \psi}, \quad \frac{\partial}{\partial e^{*}} \frac{\mathrm{d} \lambda}{\mathrm{d} \nu}=\frac{2}{\sin 2 \psi}$.

It has been assumed that $e^{*} \approx \phi^{*}$ where in fact $e^{*}=\sin \phi^{*}=$ $\cos \psi$ in the referenced work. Clearly, since $\psi \sim \pi / 2$, the pericenter is easily destabilized if the mean inclination at epoch does not correspond to the mean inclination of the equilibria.

For instance, far from the nucleus, motion under radiation pressure has a large short-periodic amplitude $\delta x$, and the probe's actual (true) elements $x$ could be quite different from its mean orbital elements $\bar{x}$, Eq. (23). In order to improve the stability of the so-called solar plane-of-sky equilibria, Eq. (46), transformations between mean and true elements for low eccentricities have therefore been derived by Mysen (2004), where $\mu_{\mathrm{c}} \equiv 1$.

To illustrate, a simulation is included where the probe is started at the (true) true anomaly $f=0$. The stable orbit's true inclination $I_{\mathrm{t}}$ is then given by

$\cos I_{\mathrm{t}}=\frac{\delta H}{G}=\frac{\bar{\xi}}{R^{2}} \frac{a^{2}}{\mu_{\mathrm{c}} \sqrt{1-e^{* 2}}}$,

corresponding to $\bar{x}^{*}$ and not $x_{0}=\bar{x}^{*}$. Figure 8 shows the simulated eccentricity under the perturbation of radiation pressure for the extreme initial conditions

$\lambda_{0}=-\pi / 2, \quad a_{0}=50 r_{\mathrm{c}}$,

leading to the initial

$e^{*}=0.06843, \quad I_{\mathrm{t}}=1.356$

at $R=4 \mathrm{AU}$. Here $I_{0}=I_{\mathrm{t}}$, representing $x_{0}=\bar{x}^{*}+\delta x_{0}$, is used for the dotted line, while the solid one represents the case $x_{0}=$ $\bar{x}^{*}$. In both cases it is seen that the probe eventually escapes, but that the use of the true equilibria (dashed line) increases the deflection angle considerably. The inclusion of tidal acceleration Eq. (4) in the simulations have no visible effects on the results of Fig. 8.

\subsection{An escape criterion}

The energy of the orbiter under the influence of radiation pressure and the nucleus' gravitational attraction can be defined according to

$F \equiv \frac{1}{2} \dot{\boldsymbol{r}}^{2}+V_{0}+V_{1}=-\frac{\mu_{\mathrm{c}}}{2 a}+\frac{\xi}{R^{2}} \boldsymbol{u}_{\odot} \cdot \boldsymbol{r}$

with the perturbation $V_{1}$ of Eq. (7). At terms with basic Hamiltonian theory one has for the time derivative of the energy

$\dot{F}=\frac{\partial F}{\partial t} \sim r$

From simulations where the solar cell arrays do not rotate with respect to the Sun we see that $\Delta F \approx 0$ as long as the probe orbit remains bound, i.e. small $r$ of Eq. (54).

For an approximately conserved $F$, escape is naturally only possible if always $F-V_{0}-V_{1}>0$ on the probe's journey outwards. The easiest way to accomplish this is in the direction where the maximum of $V=V_{0}+V_{1}<0$ is smallest, which is directly away from the Sun and at cometocentric distance (Schwinger 2001)

$r_{\mathrm{H}}=\sqrt{\frac{\mu_{\mathrm{c}}}{\xi}} R$.

Otherwise, the escape direction $\cos \theta_{\mathrm{e}}=-\boldsymbol{u}_{\odot} \cdot \boldsymbol{r} / \boldsymbol{r}$ should be defined. The smallest maximum of $V$ is then easily shown to be given by

$V_{\mathrm{H}}=-2 \sqrt{\frac{\mu_{\mathrm{c}} \xi \cos \theta_{\mathrm{e}}}{R^{2}}}$.

If the orbit is started in the solar plane-of-sky, it will escape at heliocentric distance

$F_{0}=V_{\mathrm{H}} \Longrightarrow R_{\mathrm{e}}=4 \sqrt{\cos \theta_{\mathrm{s}}} \sqrt{\frac{\xi}{\mu_{\mathrm{c}}}} a_{0}$.

Consistent with the given initial conditions, one can expect the escape angle $\theta_{\mathrm{e}}$ to be high. In fact, seen from a large number of simulations with a wide range of starting eccentricities, perturbation strengths, nucleus masses and semi-major axes, the spacecraft is usually rapidly ejected at the heliocentric distance implied by a small

$\sqrt{\cos \theta_{\mathrm{s}}} \sim 0.5$.

Shown as Fig. 9 are escape distances $R_{\mathrm{e}}(e=1)$ from a selection of these simulations with the probe starting at its apocenter and $\lambda_{0}=g_{0}=I_{0}=\pi / 2$, using different initial eccentricities, $e_{0}=0.2$ (boxed) and $e_{0}=0.7$ (cross). In addition, results from simulations where the nucleus mass is reduced to one third of its nominal value, and another one where the radiation pressure is reduced by the same factor, are also given in the plot. The predicted values, Eqs. (57) and (58), are represented by the solid lines, and one can see that they are fairly representative. Criterion (57) with (58) also works well for probes which start at the radiation pressure equilibria.

\section{Outgassing pressure}

\subsection{Model}

According to Schulz et al. (2004), "The Giotto images of comet $1 \mathrm{P} /$ Halley showed that material evaporated off certain areas of 


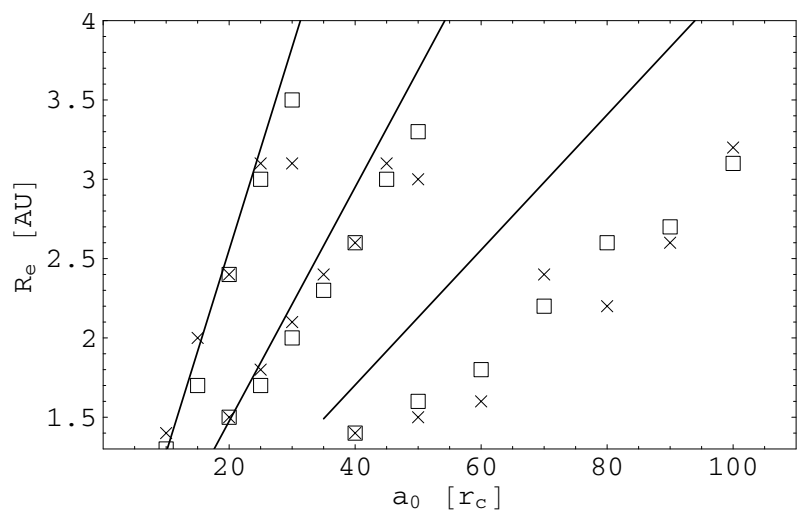

Fig. 9. Heliocentric distance of escape for a cometary orbiter under the influence of radiation pressure according to simulations (boxes and crosses) and predictions (solid lines). The steepest line is for a reduced nucleus mass, while the most slowly increasing line is for a reduced radiation pressure strength.

Halley's nucleus in the form of jets, which led to the concept of a comet nucleus being divided in active and inactive areas." If this model had not been problematic today, one could have hoped to develop fairly representative models of the interaction between the comet coma and an orbiting spacecraft, as the connection between certain coma structures and the nucleus surface would be at least conceptually simple.

However, it has been shown that discontinuous gas shocks and dust jets also arise from surface irregularities (Crifo \& Rodionov 1997b), and temperature differences along (Crifo et al. 1999) the surface. And even further, that adopting a random distribution of active and non-active regions does not affect considerably a consistent reproduction of 1P/Halley's coma by such models (Crifo et al. 2002). Or stated more clearly, "the circumnuclear coma is not related in a trivial way to the local properties of the (nuclei) surfaces." (Crifo \& Rodionov 1997b).

Nevertheless, despite the complicated conditions prevailing in cometary comae, there are two general properties which usually are adopted as representative for its interaction with the probe, illustrated by a pressure field formula of Scheeres et al. (2000) for continuous outgassing

$P_{\mathrm{c}}=\frac{P_{0}}{r^{2}} \sum_{n=0}^{N} \sum_{m=0}^{n} P_{n m}(\cos \phi)\left[\alpha_{n m} \cos m \lambda+\beta_{n m} \sin m \lambda\right]$,

$\ddot{\boldsymbol{r}}=\frac{P_{\mathrm{c}}}{\chi_{r}} \boldsymbol{u}_{r}, \quad \alpha_{00} \equiv 1$,

for the acceleration of a probe a distance $r$ from some geometrical center of the comet nucleus, clearly a subgroup of Eq. (10). As before, $\phi$ is the probe's cometocentric angular distance from the direction of the Sun, $\boldsymbol{u}_{\odot}$, and the coordinate of the pressure point is then uniquely given by some angle $\lambda$ in the plane normal to $\boldsymbol{u}_{\odot}$, see Fig. 10 . The coefficients $\alpha_{n m}$ and $\beta_{n m}$, which we later will allow to be dependent on time, are non-sphericity parameters.

Applications of Eq. (59) to simulations of the comae of the Deep Impact target 9P/Tempel 1 and the previous Rosetta comet 46P/Wirtanen, yielded good fits with constant coefficients, implying that the $\sim r^{-2}$ dependency could be a fair approximation for the background field. This should be viewed in the light of Crifo \& Rodionov (1997b), where it is concluded that although both the corotating and background distributions undergo significant spatial distortions as the comet nucleus rotates, the latter stays approximately fixed in the solar-ecliptic frame.

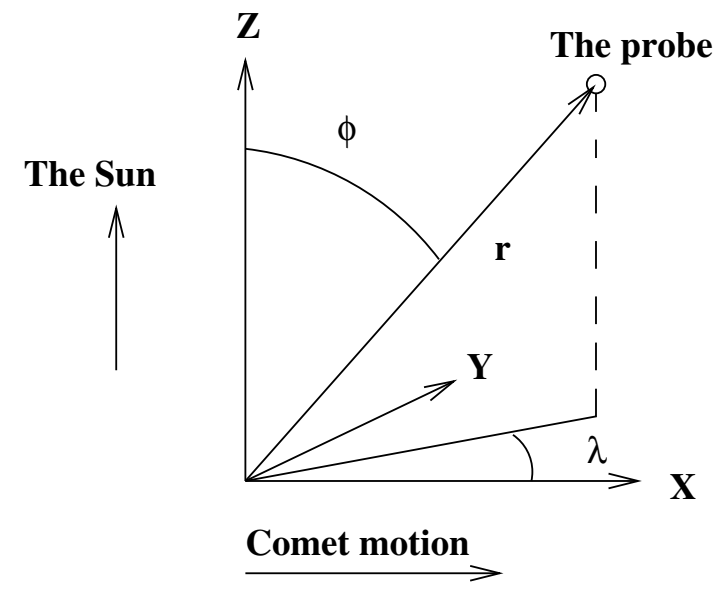

Fig. 10. The pressure point angles are given relative to a rotating reference frame. The reference $X$ - and $Y$-axes now lie in the solar plane-ofsky normal to the Sun's direction.

In Crifo \& Rodionov (1997a), the coma of the smaller previous Rosetta target 46P/Wirtanen was simulated adopting active and inactive areas, resulting in distinct shock structures between $50^{\circ} \lesssim \phi \lesssim 70^{\circ}$. Again, we extrapolated the drag factors from the plots in a cross-section of constant $\lambda$, again leading to $\varrho v^{2} \sim r^{-2}$ on a precision scale of integer $n$ 's, as from $\ddot{\boldsymbol{r}} \sim r^{-n} \boldsymbol{u}_{r}$, despite the clear shock structures. As previously mentioned, the pressure field in the $\phi=0$ direction was seen to be about an order of magnitude stronger than if the pressure would have been distributed uniformly on a sphere.

For real comets, $\alpha$ and $\beta$ most certainly change in time, and they should therefore, at least partly, be modelled as stochastic processes. Interestingly, such a scheme could be extended to absorb the more discontinuous interactions, but then naturally leading to a stronger stochastic part.

We must finally stress that we do not advocate the universality of Eq. (59)'s $\sim r^{-2}$ dependency, but merely point out that it possibly could lower significantly the tracking data residuals on Rosetta in a bound orbit around 67P/Churyumov-Gerasimenko.

\subsection{Regular dynamics}

A radial force, like the one of Eq. (59), implies that the orbiting probe's cometocentric orbital angular momentum $\boldsymbol{G}$ is conserved. That is, the orbit's longitude of ascending node $h$ and inclination $I$ are constant, as long as they are referred to non-rotating system comoving with the comet. Also, the orbit's cometocentric semi-major axis $a$ and eccentricity $e$ are linked by the conserved magnitude $G=\sqrt{\mu_{\mathrm{c}} a\left(1-e^{2}\right)}$.

As already mentioned, Eq. (59) can be taken to represent both the pressure background field and the more jet-like features of the coma if the expansion's coefficients are allowed to change in time. Except for a possible slow heliocentric modulation in strength, these explicit time dependencies will, consistent with the prerequisites of regular motion, be assumed periodic.

For a radial force, one has the following time derivatives of some remaining parameters (Murray \& Dermott 1999)

$\dot{e}=\frac{G}{\mu_{\mathrm{c}}} \frac{P_{\mathrm{c}}}{\chi_{r}} \sin f=\frac{1-e^{2}}{2 e} \frac{\dot{a}}{a}, \quad \dot{g}=-\frac{G}{\mu_{\mathrm{c}} e} \frac{P_{\mathrm{c}}}{\chi_{r}} \cos f$. 
With the use of the Legendre function expansions of Kinoshita et al. (1974), and the operator equation

$$
\int \mathrm{d} \tilde{l}=\int \mathrm{d} f\left(\frac{r}{a}\right)^{2}\left(1-e^{2}\right)^{-1 / 2},
$$

where $\tilde{l}$ and $f$ are the probe's mean and true anomaly, respectively, the averaged equations are easily worked out in terms of the so-called modified Jacobi polynomials $\bar{Q}_{n}$

$$
\begin{gathered}
\frac{1}{2 \pi} \int_{0}^{2 \pi} \mathrm{d} f \sin f P_{n m}(\cos \phi) \exp [\mathrm{i} m \lambda]=-\sum_{\varepsilon} P_{n 1}(0) \\
\quad \times \bar{Q}_{n}^{(m, 1)}(\varepsilon, \cos \tilde{I}) \frac{\varepsilon}{2}(-\mathrm{i})^{m} \exp [\mathrm{i}\{\varepsilon \tilde{g}+m \tilde{h}\}]
\end{gathered}
$$

and

$$
\begin{gathered}
\frac{1}{2 \pi} \int_{0}^{2 \pi} \mathrm{d} f \cos f P_{n m}(\cos \phi) \exp [\mathrm{i} m \lambda]=\sum_{\varepsilon} P_{n 1}(0) \\
\quad \times \bar{Q}_{n}^{(m, 1)}(\varepsilon, \cos \tilde{I}) \frac{\mathrm{i}}{2}(-\mathrm{i})^{m} \exp [\mathrm{i}\{\varepsilon \tilde{g}+m \tilde{h}\}]
\end{gathered}
$$

where $\varepsilon$ here is an index taking on the values +1 and -1 , and $\mathrm{i}$ is the complex number directed along the imaginary axis, with norm equal to one. The angles $\tilde{I}, \tilde{h}$ and $\tilde{g}$ above are the orbit's inclination, longitude of ascending node and argument of pericenter, respectively, of the probe relative to the solar plane-ofsky, see Fig. 10. One should note that these are different from the type $g$ of Eq. (60), which is referred to an arbitrary comet comoving, but non-rotating reference system, see Fig. 6. It is of interest that the inclination and node in the rotating system are not dependent on the dynamical variables, i.e.

$\tilde{I}=\tilde{I}\left(\tilde{h}_{0}, \tilde{I}_{0}, t\right), \quad \tilde{h}=\tilde{h}\left(\tilde{h}_{0}, \tilde{I}_{0}, t\right)$.

The equations for the averaged system are now

$\dot{e}=-\frac{P_{0}}{\bar{\chi}_{r}} \frac{1}{\sqrt{\mu_{\mathrm{c}} a^{3}}} \sum_{n=1}^{N} \sum_{m=1}^{n} \sum_{\varepsilon} \varepsilon \operatorname{Re}\left[\Xi_{n m}\right]$

$\dot{g}=-\frac{P_{0}}{\bar{\chi}_{r}} \frac{1}{\sqrt{\mu_{\mathrm{c}} a^{3}} e} \sum_{n=1}^{N} \sum_{m=1}^{n} \sum_{\varepsilon} \operatorname{Re}\left[\mathrm{i} \Xi_{n m}\right]$,

where

$$
\begin{aligned}
\Xi_{n m}= & \frac{P_{n 1}(0)}{2} \bar{Q}_{n}^{(m, 1)}(\varepsilon, \cos \tilde{I})(-\mathrm{i})^{m} \\
& \times \sqrt{\bar{\alpha}_{n m}^{2}+\bar{\beta}_{n m}^{2}} \exp \left[\mathrm{i}\left\{\varepsilon \tilde{g}+m \tilde{h}-\varphi_{n m}\right\}\right]
\end{aligned}
$$

and

$\cos \varphi_{n m}=\frac{\bar{\alpha}_{n m}}{\sqrt{\bar{\alpha}_{n m}^{2}+\bar{\beta}_{n m}^{2}}}$.

It is important to recognize that

$\dot{a} \sim e\left(\mu_{\mathrm{c}} a\right)^{-1 / 2}$

drops slowly with increasing orbital radius, and that only the odd $n$ harmonics contribute to the secular rate since $P_{n 1}(0)=0$ for $n$ even. That is, the stationary part of the pressure field must have an antisymmetry with respect to the solar plane-of-sky if orbital changes are to take place, a scenario which is certain to occur. For Eqs. (65) and (66) to be valid it is assumed that the variation of the probe's exposed area and the pressure field coefficients etc. do not "beat" with the mean anomaly of the orbiter

$\left|\boldsymbol{S}_{\mathrm{S} / \mathrm{C}} \cdot \boldsymbol{u}_{r}\right| \nsim \cos (\tilde{l}+\varphi)$,

i.e. that their Fourier expansions are not dominated by frequencies close to the probe's orbital frequency, or integer multiples of it for that matter. Condition Eq. (70) coincides with the nonresonance prerequisite in Hamiltonian systems.

\subsection{Solar plane-of-sky}

Now, if we define $\tilde{X} \equiv \sqrt{a} \mathcal{X}$, which is approximately secularly conserved under the influence of radiation pressure, it can easily be concluded that radial outgassing does not compromise this quantity's constancy, and therefore that the orbit plane is still secularly constrained to the vicinity of the solar plane-of-sky if the probe is started there. However, if the semi-major axis of the orbit increases, the more loose these constraints become, and the maximum eccentricity grows

$1-e_{\mathrm{M}}^{2} \approx a^{-1} \tilde{X}_{0}^{2}$.

Below, the possibilities and consequences of such a scenario are studied.

Further simplifications of Eqs. (65) and (66) can be made for the case $\tilde{I}=0$, corresponding to a mean orbit normal $\boldsymbol{n}_{\mathrm{o}}$ along $\boldsymbol{u}_{\odot}$,

$\bar{Q}_{n}^{\left(m, m^{\prime}\right)}(\varepsilon, 1)=\delta_{m, m^{\prime}}, \quad \varepsilon=+1, \quad g=\tilde{g}-\pi$

if $\tilde{h} \equiv 0$ which we are free to do. Likewise, for an orbit normal along $\boldsymbol{n}_{o}=-\boldsymbol{u}_{\odot}(\tilde{I}=\pi)$,

$\bar{Q}_{n}^{\left(m, m^{\prime}\right)}(\varepsilon,-1)=\delta_{m, m^{\prime}}, \quad \varepsilon=-1, \quad g=\tilde{g}$,

resulting in the equations

$\dot{e}=-\sum_{n=1}^{N} \mathcal{A}_{n} \sin u_{n}, \quad \dot{\tilde{g}}=-\sum_{n=1}^{N} \frac{\mathcal{A}_{n}}{e} \cos u_{n}$

where

$\mathcal{A}_{n}=\frac{P_{0}}{\bar{\chi}_{r}} \frac{1}{\sqrt{\mu_{\mathrm{c}} a^{3}}} \frac{P_{n 1}(0)}{2} \sqrt{\bar{\alpha}_{n 1}^{2}+\bar{\beta}_{n 1}^{2}}$

and

$u_{n}=\tilde{g} \pm \varphi_{n 1}, \quad \boldsymbol{n}_{o}=\mp \boldsymbol{u}_{\odot}$.

These equations are tested with a few simulations, also in order to illustrate typical associated secular changes. The perturbation strength will correspond to the roughly nominal CO outgassing rate and a minimum $\chi_{r}$

$\frac{P_{0}}{\bar{\chi}_{r}}($ nominal $)=0.01 r_{\mathrm{c}}^{3} \mathrm{~h}^{-2}$.

The only other coefficients of the expansion Eq. (59) which here, for convenience, are defined as non-zero, are set to

$\bar{\alpha}_{10}=0.9, \quad \bar{\alpha}_{11}=0.4, \quad \bar{\beta}_{11}=0.1, \quad \varphi_{11}=0.245$,

differing from those used in the simulation by a periodic component $\sim \sin \left(2 \pi P_{\mathrm{c}}^{-1} t+\varphi\right)$ with amplitude in the same order of magnitude as the stationary part. The initial conditions are

$\tilde{I}_{0}=0, \pi, \quad g_{0}=\pi / 2, \quad a_{0}=10 r_{\mathrm{c}}, \quad e_{0}=0.2$. 


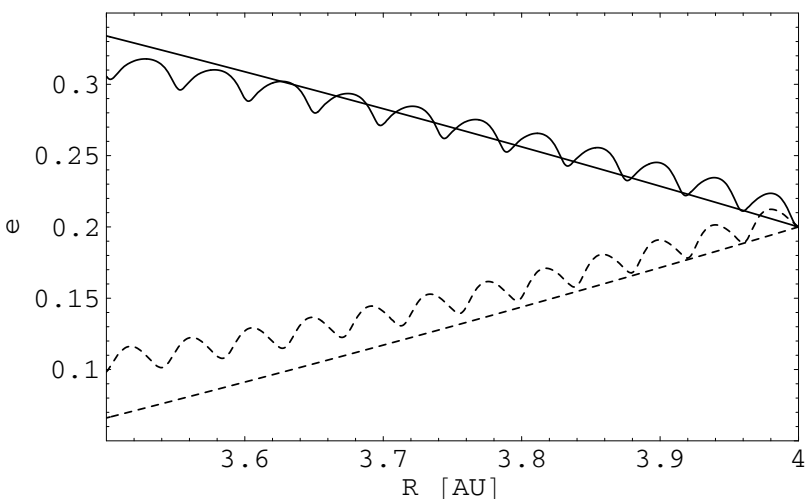

Fig. 11. The outgassing induced variation of the orbiting probe's eccentricity as a function of the comet's preperihelion distance. The initial condition $\tilde{I}_{0}=0$ is represented by the solid line, while the dashed one has $\tilde{I}_{0}=\pi$. The linear extrapolation represents the analytical prediction.

Figure 11 shows the eccentricities based on the simulations together with their analytical predictions as inferred from Eq. (74). As one clearly can see, the predictions are good, taking into account that the rates have been calculated using instantaneous rather than mean epoch elements as they should. The limitations of a linear secular approach to the motion are also evident.

As recognized by Scheeres et al. (1998a), the existence of radiation pressure equilibria Eqs. (46), is not affected by an outgassing pressure field which is symmetric around $\boldsymbol{u}_{\odot}$ since the index $m$ of these components is zero. However, for a cometocentric field which "remembers" the direction of comet motion, $\alpha \neq 0$, and the stability of the equilibria could be questioned. Indeed, if, for simplicity one makes the fair assumption that the first components which appear in expansion Eq. (59) are the strongest, motion under Eqs. (74) alone seeks the asymptotic behaviour (indexed $a$ )

$\tilde{g}_{a} \pm \varphi_{11}=-\pi / 2, \quad \dot{e}_{a}=\mathcal{A}_{1}=\frac{1-e_{a}^{2}}{2 e_{a}} \frac{\dot{a}_{a}}{a_{a}}>0$,

which coincides with the radiation pressure equilibria if $\alpha>0$ and $\beta=0$, i.e. if the pressure field in the solar plane-of-sky is strongest in the direction of comet motion. As a result, the semi-major axis and the maximum eccentricity, Eq. (71), will grow. Choosing the initial eccentricity to be low on the other hand, minimizes the secular rate of the orbital energy and, selfconsistently, $e_{\mathrm{M}}$ stays small if the outgassing pressure is not too strong.

That the eccentricity stays small is important since the outgassing's ability to alter the cometary orbiter's energy increases with orbit ellipticity. As in Mysen \& Aksnes (2005) the outgassing caused change in energy, Eq. (53), on an orbital leg from $r_{1}$ to $r_{2}$ is easily shown to be

$\Delta F=\int_{t_{1}}^{t_{2}} \mathrm{~d} t \frac{P_{\mathrm{c}}}{\chi_{r}} \frac{\boldsymbol{r}}{r^{3}} \cdot \dot{\boldsymbol{r}} \approx \overline{P_{0 \chi_{r}^{-1}}}\left(r_{1}^{-1}-r_{2}^{-1}\right)$

where the bar indicates a mean along the orbit. Under the assumption that the energy otherwise is conserved, the condition $F=V_{0}+\Sigma \Delta F=V_{\mathrm{H}}$ indicates escape.

Included as Fig. 12 is the energy $F$ from a full simulation of an orbiter's motion under the influence of radiation pressure and a worst-case $\mathrm{CO}$ outgassing field

$\frac{P_{0}}{\bar{\chi}_{r}}($ worst-case $)=0.1 r_{\mathrm{c}}^{3} \mathrm{~h}^{-2}$

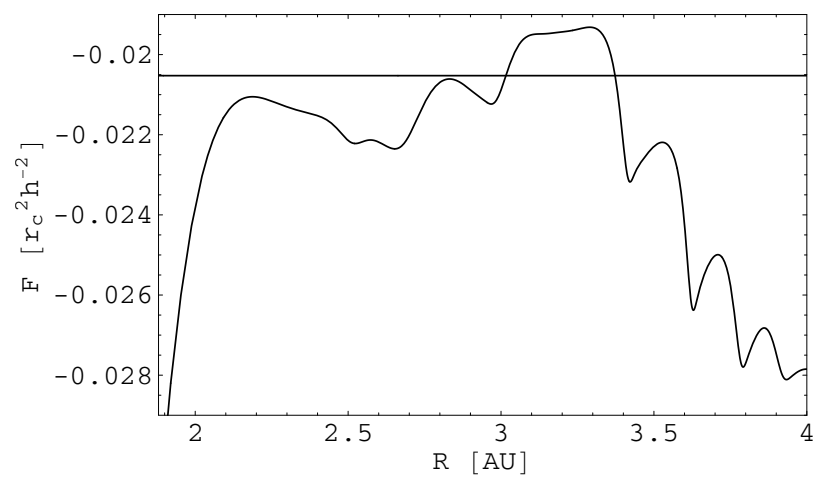

Fig. 12. The evolution of an orbiter's energy $F$ under a worst-case $\mathrm{CO}$ pressure field and full solar cell array exposure to radiation. The value of the potential $V_{\mathrm{H}}$ Eq. (56) at the seen escape distance $R_{\mathrm{e}}=2.0 \mathrm{AU}$ is represented by the bar.

The stationary coefficients of the field are set to the same values as Eqs. (78), and the probe is started in a solar plane-of-sky equilibrium in order to minimize $e_{\mathrm{M}}$, with $a_{0}=20 r_{\mathrm{c}}$. Although the energy has its maximum before $R=3 \mathrm{AU}$, radiation pressure is too weak to eject the probe at this heliocentric distance. Consistent with our adopted criterion, the probe escapes when $V_{\mathrm{H}}$ of Eq. (56), represented by the solid line for the observed escape distance, and $F$ converge to the same value. With the use of Eq. (81) one can estimate the number of orbits before the probe is ejected.

From these considerations, or more directly from the fact that $a$ and $e \rightarrow \infty$ rapidly for strong outgassing, it should now be evident that the outgassing equilibrium Eq. (80) should be avoided. One way to accomplish this is to actively control the probe orbit's pericenter if possible so that $u_{1} \equiv 0$ or $\pi / 2$. However, if the asymmetries of the pressure field are different from $\alpha>|\beta|>0$, significant stability can be achieved with a proper choice of pericenter. That is, if $\pi / 2 \lesssim \varphi_{11} \lesssim \pi$, the radiation pressure equilibria compete with the outgassing driven asymptotic behaviour Eq. (80) as a stable orbit configuration. As a result, the secular rate of the semi-major axis is not necessarily strictly positive over time. These concepts are clearly shown in Fig. 13 where the same orbital parameters of the simulation shown in Fig. 12 are used, except that $a_{0}=30 r_{\mathrm{c}}$ and $\bar{\alpha}_{11}= \pm 0.4$. For $\cos \varphi_{11}>0$ (solid), the radiation pressure and outgassing equilibria are nearly aligned and the probe is relatively quickly ejected at about $R=2.5 \mathrm{AU}$. In the case that $\cos \varphi_{11}<0$ (dashed), the equilibria under the different effects are almost completely non-aligned, and the orbiter's stability is actually improved in comparison to the pure radiation pressure problem due to the resulting unstable evolution of the pericenter. In the limit of a weaker outgassing field, Eq. (77), the two asymmetry scenarios converge to the outgassing free problem, Eqs. (57) and (58), as illustrated in Fig. 14. Again, the probe is started in a radiation pressure equilibrium orbit, but now the outgassing strength corresponds to that of Eq. (77), and the nucleus mass is set to one fifth of our adopted nominal value.

It is important to note that if the radiation pressure induced change in pericenter $\dot{g}_{\odot}$ is smaller than that generally caused by outgassing $\dot{g}_{c}$, the asymptotic motion Eqs. (80) will again dominate independently of equilibria alignment

$$
\begin{aligned}
\dot{g}_{\odot}=\frac{\partial \bar{V}_{1}}{\partial G} \sim & \frac{3}{2} \frac{\bar{\xi}}{R^{2}} \sqrt{\frac{a}{\mu_{\mathrm{c}}}} \frac{1}{e} \\
& <\dot{g}_{\mathrm{c}} \sim \frac{1}{2} \frac{P_{0}}{\bar{\chi}_{r}} \frac{1}{\sqrt{\mu_{\mathrm{c}} a^{3}}} \frac{1}{e} \sqrt{\bar{\alpha}_{11}^{2}+\bar{\beta}_{11}^{2}}
\end{aligned}
$$




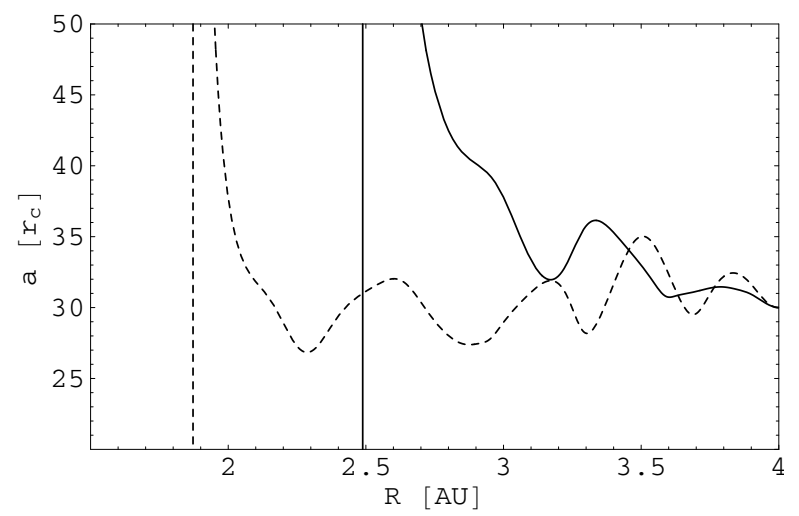

Fig. 13. Evolution of a cometary orbiter's semi-major axis under the influence of a strong $\mathrm{CO}$ pressure field and full solar cell array exposure to radiation. The solid curve represents the case $\cos \varphi_{11}>0$, while $\cos \varphi_{11}<0$ is represented by the dashed one.

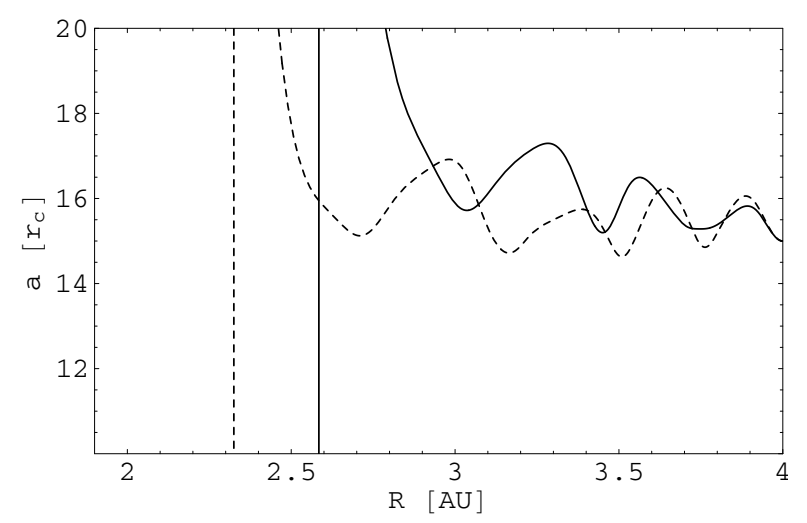

Fig. 14. The semi-major axis evolution under the influence of a nominal $\mathrm{CO}$ pressure field, low nucleus mass and full solar cell array exposure to radiation. The angle $\cos \varphi_{11}>0$ is represented by the solid curve, while the dashed one is for $\cos \varphi_{11}<0$.

or

$a<a_{\mathrm{c}} \equiv \sqrt{\frac{1}{3} \frac{R^{2}}{\bar{\xi}} \frac{P_{0}}{\bar{\chi}_{r}}\left(\bar{\alpha}_{11}^{2}+\bar{\beta}_{11}^{2}\right)^{1 / 2}} \sim 3 \frac{R}{\mathrm{AU}} r_{\mathrm{c}}$

where the tabulated value is for nominal nucleus mass, full solar cell array exposure to the Sun and the outgassing pressure strength Eq. (82) with parameters Eqs. (78).

The paper will be concluded with two more simulations where the runs illustrated in Fig. 13 are repeated, but with a $a_{0}=15 r_{\mathrm{c}}$ and the very large

$\frac{P_{0}}{\bar{\chi}_{r}}($ water $)=1 r_{\mathrm{c}}^{3} \mathrm{~h}^{-2}\left(\frac{1.3 \mathrm{AU}}{R}\right)^{2}$,

its perihelion strength roughly corresponding to, for instance, maximum spacecraft area exposure to the outgassing, and the water outgassing pressure of Fig. 5 in the solar plane-of-sky if the field strength is reduced an order of magnitude from $\phi=0$ to $\phi=\pi / 2$, as can be seen from the simulations of Crifo \& Rodionov (1997a). The results are given in Fig. 15, and show that bound orbits through the comet's perihelion are not possible in such a scenario without active control, even though the pressure field asymmetries are favourable. If, on the other hand, the area exposed to outgassing is minimized, a beneficial asymmetry can be expected to make a bound orbit through the perihelion possible for $a_{0} \lesssim 15 r_{\mathrm{c}}$, as is verified by simulations.

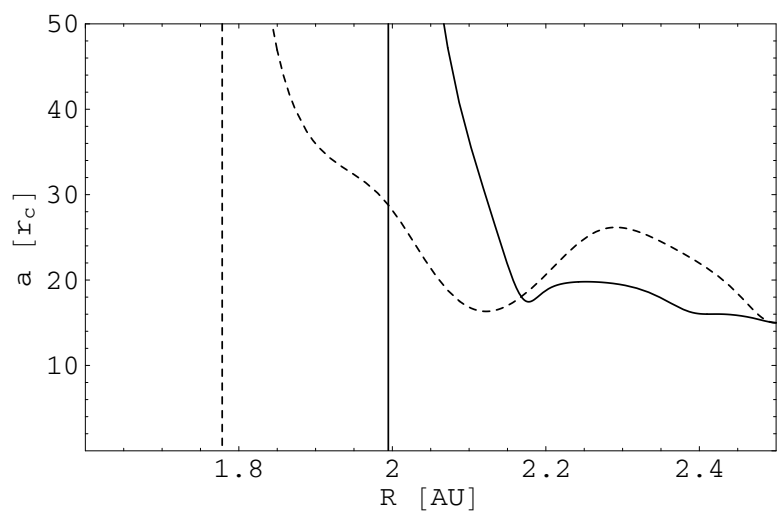

Fig. 15. A cometary orbiter's semi-major axis evolution under the influence of a strong water pressure field and maximum exposure of the solar cell arrays to radiation. The solid curve represents the case $\cos \varphi_{11}>0$, while $\cos \varphi_{11}<0$ is represented by the dashed one.

\section{Discussion}

The perturbation strengths of gravity, radiation and outgassing pressure on a bound Rosetta orbit around target comet 67P/Churyumov-Gerasimenko have been evaluated. In what we have adopted as a nominal scenario, where the $\mathrm{CO}$ outgassing pressure was set to one tenth of its upper limit, it was found that the perturbations can be considered weak and motion regular a long time after rendezvous. It is pointed out that the rotationally modulated part of the gravity field of the comet nucleus almost does not affect the secular stability of these orbits' shape at all, and that such radiation pressure induced instabilities are minimized if the initial orbit is chosen to have low eccentricity, and to lie in the solar plane-of-sky. The comet heliocentric distance of probe escape from an orbit which is started in this plane normal to the cometocentric direction of the Sun, is seen to be dependent on the spacecraft orbit's initial semi-major axis only, and linearly so.

For the calculation of the outgassing induced secular changes of the cometary orbiter's cometocentric orbital elements, an asymmetric and periodically time-varying outgassing pressure field is adopted. If the incident molecules are thermalized and reflected diffusely by the probe, a range of spacecraft orientations with respect to the gas flow, dependent on the variations of the probe temperature across its surface, results in a force directed mainly away from the nucleus. Accordingly, the asymptotic tendency of the outgassing is, in the solar plane-of-sky, to eject the probe from a bound orbit. For a pressure field which in the solar plane-of-sky is strongest in the direction opposite to the comet's motion, the radiation pressure equilibria destabilize this outgassing driven asymptotic behaviour. If the field is strongest in the direction of comet motion, the two equilibria of the different effects are aligned and the probe escapes rapidly.

For full solar cell array exposure to radiation, orbits in the solar plane-of-sky with semi-major axis $a \lesssim 15 r_{\mathrm{c}}$ could remain passively bound through the comet's perihelion if the pressure field asymmetries are favourable, the nucleus mass nominal and the outgassing exposed area minimized.

At last, we stress that issues related to the modelling of the outgassing pressure field must be addressed in a more systematic way. For instance, to what extent is the inverse square law representable? What are the frequencies and related amplitudes of the pressure field's temporal variations? More precisely, to what extent is the flow radial, the coma of low temperature and the mean free path of the molecules much larger than the spacecraft? 
These questions depend on the numerous uncertain parameters of the problem, and can possibly therefore only be answered to a limited extent, and then only by the most advanced codes today. Future treatments should incorporate effects from aerodynamic lift.

Acknowledgements. This work was financed by the Research Council of Norway, project 153382 and 170870.

\section{References}

Anderson, J. D., Lau, E. L., Bird, M. K., et al. 2004, J. Geophys. Res., 109 Biver, N., Bockelée-Morvan, D., Colom, P., et al. 1997, Earth, Moon and Planets 78,5

Bockelée-Morvan, D., Moreno, R., Biver, N., et al. 2004, in The New ROSETTA Targets, ed. L. Colangeli, E. M. Epifani, \& P. Palumbo (Dordrecht: Kluwer), ASSL, 311, 25

Capria, M. T., Coradini, A., De Sanctis, M. C., et al. 2004, in The New ROSETTA Targets, ed. L. Colangeli, E. M. Epifani, \& P. Palumbo (Dordrecht: Kluwer), ASSL, 311, 177

Cook, G. E. 1965, Planet. Space Sci., 13, 929

Crifo, J. F. 1987, A\&A, 187, 438

Crifo, J. F., \& Rodionov, A. V. 1997a, Icarus, 127, 319

Crifo, J. F., \& Rodionov, A. V. 1997b, Icarus, 129, 72

Crifo, J. F., Itkin, A. L., \& Rodionov, A. V. 1995, Icarus, 116, 77

Crifo, J. F., Rodionov, A. V., \& Bockelée-Morvan, D. 1999, Icarus, 138, 85

Crifo, J. F., Rodionov, A. V., Szegö, K., et al. 2002, Earth, Moon and Planets, 90, 227

Crifo, J. F., Loukianov, G. A., Rodionov, A. V., et al. 2003, Icarus, 163, 479

Crifo, J. F., Lukyanov, G. A., Zakharov, V. V., et al. 2004, in The New ROSETTA Targets, ed. L. Colangeli, E. M. Epifani, \& P. Palumbo (Dordrecht: Kluwer), ASSL, 311, 119

Davidsson, B. J. R., \& Gutiérrez, P. J. 2005, Icarus, 176, 453

De Sanctis, M. C., Capria, M. T., \& Coradini, A. 2005, A\&A, 444, 605

Enzian, A., Klinger, J., Schwehm, G., et al. 1999, Icarus, 138, 74

Fulle, M., Barbieri, C., Cremonese, G., et al. 2004, A\&A, 422, 357

Goldstein, H. 1980, Classical Mechanics (Reading: Addison-Wesley)

Gunnarsson, M., Rickman, H., Festou, M. C., et al. 2002, Icarus, 157, 309

Heiskanen, W. A., \& Moritz, H. 1967, Physical Geodesy (San Francisco: W.H. Freeman and Company)

Hori, G. 1966, PASJ, 18, 297

King-Hele, D. G. 1987, Satellite Orbits in an Atmosphere: Theory and Applications (Glasgow: Blackie and Son Ltd)
Kinoshita, H. 1972, PASJ, 24, 423

Kinoshita, H., Hori, G., \& Nakai, H. 1974. Modified Jacobi polynomials and its applications to expansions of disturbing functions, Annals of the Tokyo Astronomical Observatory, Second Series, Vol. XIV N.1

Kovalevsky, J. 1967, Introduction to Celestial Mechanics (Dordrecht: D. Reidel Publishing Company), ASSL, 7

Królikowska, M. 2003, Acta Astron., 53, 195

Lamy, P. L., Toth, I., Weaver, H., et al. 2003, The nucleus of comet 67P/Churyumov-Gerasimenko, the new target of the Rosetta mission, DPS 35th Meeting

Mignard, F. 1982, Icarus, 49, 347

Mignard, F., \& Henon, M. 1984, Cel. Mech., 33, 239

Montenbruck, O., \& Gill, E. 2000, Satellite Orbits (Germany: Springer-Verlag)

Murray, C. D., \& Dermott, S. F. 1999, Solar System Dynamics (Cambridge: Cambridge University Press)

Mysen, E. 2004, in The New ROSETTA Targets, ed. L. Colangeli, E. M. Epifani, \& P. Palumbo (Dordrecht: Kluwer), ASSL, 311, 211

Mysen, E. 2005, Content and sensitivity analysis of radiometric signals generated by a deep space orbiter, IAUS 229 - ACM 2005

Mysen, E., \& Aksnes, K. 2005, The Jacobi constant for a cometary orbiter, A\&A, 443, 691

Oertzen, J. von 2003, Global modeling of comets: Nucleus, neutral and ionized coma of comets 67P/Churyumov-Gerasimenko and 46P/Wirtanen: preparations for the ROSETTA Radio Science Investigations, Ph.D. Thesis, University of Cologne

Pätzold, M., Häusler, B., Wennmacher, A., et al. 2001, A\&A, 375, 651

Rickman, H., Kamél, L., Festou, M. C., et al. 1987, ESA SP-278, 471

Rodionov, A. V., Crifo, J.-F., Szegö, K., et al. 2002, Planet. Space Sci., 50, 983

Roy, A. E. 1998, Orbital Motion (Bristol: Institute of Physics Publishing)

Scheeres, D. J. 1999, J. Astronaut. Sci., 47, 25

Scheeres, D. J., \& Marzari, F. 2002, J. Astronaut. Sci., 50, 35

Scheeres, D. J., Marzari, F., Tomasella, L., et al. 1998a, Planet. Space Sci., 46, 649

Scheeres, D. J., Ostro, S. J., Hudson, R. S., et al. 1998b, Icarus, 132, 53

Scheeres, D. J., Bhargava, S., \& Enzian, A. 2000, A Navigation Model of the Continous Outgassing Field Around a Comet, TMO Progress Report 42-142

Schulz, R., Stüwe, J. A., \& Böhnhardt, H. 2004, in The New ROSETTA Targets, ed. L. Colangeli, E. M. Epifani, \& P. Palumbo (Dordrecht: Kluwer), ASSL, 311,15

Schwinger, J. 2001, Simulation von Umlaufbahnen um einen Kometenkern, Diplomarbeit, Universität zu Köln

Teemu, J., \& Mäkinen, T. 2004, in The New ROSETTA Targets, ed. L. Colangeli, E. M. Epifani, \& P. Palumbo (Dordrecht: Kluwer), ASSL, 311, 61

Valorge, C. 1995, in Spaceflight Dynamics Part I, ed. J.-P. Carrou, CNES, 217 